\title{
AN EFFICIENT FINITE ELEMENT APPROACH FOR REDUCTION OF STRUC- TURAL VIBRATION AND ACOUSTIC RADIATION BY PASSIVE SHUNTED PIE- ZOELECTRIC SYSTEMS
}

\author{
L.P. da Silva ${ }^{1}$, J.-F. Deiü ${ }^{1}$, W. Larbi ${ }^{1}$, M.A. Trindade ${ }^{2}$ \\ ${ }^{1}$ Structural Mechanics and Coupled System Laboratory, Conservatoire National des Arts et \\ Métiers, Paris (Luciano.pereira_da_silva@cnam.fr) \\ ${ }^{2}$ Department of Mechanical Engineering, São Carlos School of Engineering at the University \\ of São Paulo
}

\begin{abstract}
The present work concerns the numerical modeling of noise and vibration reduction of thin radiating structures in the low frequency range by using shunted piezoelectric elements. The aim is to propose an efficient approach able to predict the structures most radiating vibration modes and to attenuate these modes by using piezoelectric patches bonded on the structure and connected to resistive or resonant shunt. The first step is to estimate the sound power radiated by the structure and determine the vibration modes to be controlled. In a second step, an original finite element formulation, adapted to any elastic structures with surface-mounted piezoelectric patches, is proposed to solve the electromechanical problem. Finally, numerical examples are presented in order to validate and analyze our approach.
\end{abstract}

Keywords: Vibration and sound reduction, Piezoelectric shunt, Finite element method.

\section{INTRODUCTION}

The use of piezoelectric materials for vibration damping and noise suppression of flexible structures, both in active control [3, 6, 9, 21] and in passive control [4, 7-8, 12-13, 15-24], is widely discussed in the literature. These materials, which can be used as sensors or actuators, or even both simultaneously, enable the transformation of mechanical energy into electric energy (direct piezoelectric effect) and vice-versa (indirect piezoelectric effect). Also, they are well adapted to distributed or localized control of structural vibrations and sound radiation since they are produced as very thin patches that can be embedded in composite structure and allow direct connection with an input/output electrical signal [23].

Due to the direct piezoelectric effect, a portion of the mechanical energy associated with the vibration can be transformed into electric energy and dissipated through a shunt circuit that compounds a mechanism of passive damping. A detailed description of the use of shunt circuit and piezoelectric devices can be found in the pioneer work of Hagood and von Flotow [12]. In that work, the expression for the mechanical impedance introduced by the 
piezoelectric element connected to various type of shunt circuit coupled to mechanical systems is obtained. Two main electric circuits designs composed by a simple resistor (resistive shunt) and a resistor in series with an inductor (resonant shunt) are proposed. Both of those designs connected to some piezoelectric patches produce a passive damping system for a single vibration mode, so that the electric circuit parameters, as well as the piezoelectric patches dimensions and location, have to be chosen in relation to a particular structure's eigenmode $[4,12,15,17-18,20]$. Many studies have also been focused on optimizing the shunt circuits including resistances, inductances and capacitances in series and/or parallel to produce passive damping systems for single and/or multi-mode. Examples and comparison between different types of shunt circuits can be found in [4, 7, 13, 19, 23-24].

The present work concerns the numerical modeling of noise and vibration reduction of thin structures in the low frequency range by using shunted piezoelectric elements. The aim is to propose an efficient approach able to predict the structures most radiating vibration modes and to attenuate these modes by using piezoelectric patches bonded on the structure and connected to resistive or resonant shunt circuits.

The first step is to estimate the sound power radiated by the structure (without piezo patches) and determine the vibration modes to be controlled. This is done by using the elemental radiators decomposition described in previous works $[3,6,9,16]$. This method, which assumes that the sound radiation is due to a number of discrete pistons in harmonic oscillation, can be applied to any plane surface in an infinite baffle. It only requires the knowledge of the surface geometry, the characteristics of the fluid and the velocity field distribution. In this work, a finite element approach is used to evaluate this velocity field by using a sufficient number of discrete radiating elements according to the smallest wavelength to be observed.

In a second step, an original finite element formulation [18], adapted to any elastic structures with surface-mounted piezoelectric patches, is proposed to solve the electromechanical problem. This formulation, with only one couple of electrical variables per patch, is well adapted to practical applications due to the fact that realistic electrical boundary conditions, such as equipotentiality on the electrodes, naturally appear. The global variables are also intrinsically adapted to include any external electrical circuit into the electromechanical problem and to simulate the effect of shunt damping techniques. A reduced order model of the problem is then introduced, by means of a modal expansion. It is shown that the choice of modal basis used to perform the electromechanical reduced order model depends of the shunted circuit type. Therefore, the modal basis of the short-circuit problem is the most appropriated for the resistive shunt case, and for the resonant shunt case, the modal basis of the coupled electromechanical problem seems to be the best choice.

Finally, numerical examples are presented in order to validate and analyze our approach. First, the developed electromechanical model is validated by comparison with a full 3D model (with one electrical degree-of-freedom per node) showing that the reduced order model is capable of capturing the main characteristics of the system dynamic behavior, notably in terms of attenuation. Then, sensitivity analyses concerning the piezoelectric patch (size and position) are performed in order to highlight the role of the modal electromechanical coupling factor (MEMCF) on the optimization procedure of the shunt damping techniques for the reduction of sound radiation. 


\section{ACOUSTIC RADIATION FROM PLANAR THIN STRUCTURES}

This section is devoted to the acoustic radiation properties of baffled thin planar structures. First, the vibroacoustic problem considered in the present work is introduced. Then, the elemental radiators approach is recalled.

\subsection{Vibroacoustic Problem}

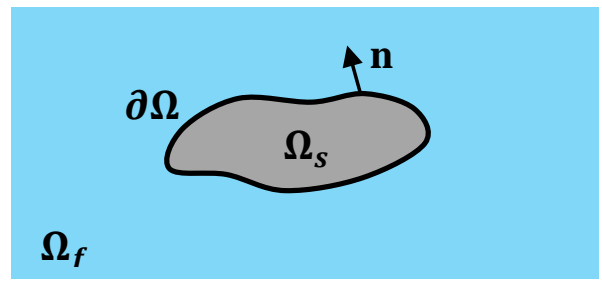

Figure 1. Vibroacoustic problem

We consider the case of an elastic structure (domain $\Omega_{s}$ ) immersed in an unbounded acoustic fluid (domain $\Omega_{f}$ ) as show in Fig. 1. The fluid-structure boundary, denoted $\partial \Omega$, is such that $\partial \Omega=\Omega_{s} \cap \Omega_{f}$. Considering weak fluid-structure interaction (light fluid medium and low frequency domain), elastic and acoustic problems can be solve independently. As a result, the acoustic problem consists in solving the following system [10]:

$$
\left\{\begin{array}{c}
\left(\Delta+K^{2}\right) \hat{p}=0 \text { in } \Omega_{f} \\
\frac{\partial \hat{p}}{\partial n}=-j \omega \rho_{f} \hat{\mathbf{v}} \mathbf{n} \quad \text { on } \partial \Omega \\
\lim _{r \rightarrow \infty}\left(r\left(\frac{\partial \hat{p}}{\partial r}+j K \hat{p}\right)\right)=0
\end{array}\right.
$$

in which the subscript $\hat{*}$ denotes a quantity in frequency domain, $p$ is the unknown pressure wave, $\mathbf{v}$ is the velocity field vector, $\mathbf{n}$ is the normal unit vector of the surface $\partial \Omega, K$ is the wave number expressed as $\omega / c_{f}$ ( $\omega$ being the angular frequency and $c_{f}$ the speed of sound in the acoustic medium), $\Delta$ is the Laplace operator, $j=\sqrt{-1}$ and $\rho_{f}$ is the fluid density. The first equation corresponds to the three-dimensional Helmholtz equation, the second is the boundary condition at the fluid-structure interface, and the last one is the Sommerfeld radiation condition (in spherical coordinates system).

The present work aims to study sound radiation in a half free space by baffled thin planar structures. In this case, the above problem system can be solving analytically by using the Spatial Fourier Transformer Method [10], the elemental radiators approach [3, 6, 9, 16], or the Rayleigh Integral $[6,9-10,25]$ which is done by:

$$
\hat{p}(M, \omega)=\rho_{f} \frac{j \omega}{2 \pi} \int_{S} \hat{\mathbf{v}}(Q, \omega) \mathbf{n}(Q) \frac{e^{-j K r}}{r} d S
$$

where $M$ is a point inside the volume $\Omega_{f}, Q$ is a point on the surface $S$ and $r$ denotes the distance between $M$ and $Q$. 
The elemental radiators method that is used in the numerical simulations of this paper will be described in the next section.

\subsection{Elemental Radiators Method}

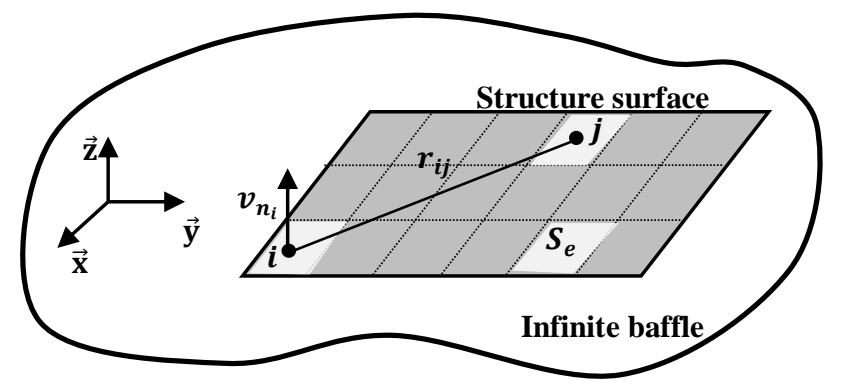

Figure 2. Principle of elemental radiators approach [6].

In the elemental radiators approach, the sound radiation is due to $N$ pistons of equal size, as showing in Fig. 2. It is assumed that the velocity and pressure fields across each radiator are constant once they are small compared with the acoustic wavelength. Then, the acoustic power radiated reduces to the summation:

$$
\Pi=\frac{S_{e}}{2} \mathfrak{R}\left(\hat{\mathbf{v}}_{\mathbf{n}}^{\mathbf{H}} \widehat{\mathbf{p}}\right)
$$

where $\widehat{\mathbf{p}}$ and $\hat{\mathbf{v}}_{\mathbf{n}}$ are the vectors of the surface complex acoustic pressure and normal volume velocity of the elementary radiators, $S_{e}$ is the surface of an elementary radiator, and subscript $\mathrm{H}$ denotes the Hermitian transpose. Using the relation $\widehat{\mathbf{p}}=\mathbf{Z} \hat{\mathbf{v}}_{\mathbf{n}}$ (Z being the matrix of acoustic impedances) the acoustic power radiated may be written:

$$
\Pi=\frac{s_{e}}{2} \mathfrak{R}\left(\hat{\mathbf{v}}_{\mathbf{n}}^{\mathbf{H}} \mathbf{Z} \hat{\mathbf{v}}_{\mathbf{n}}\right)=\frac{s_{e}}{4} \hat{\mathbf{v}}_{\mathbf{n}}^{\mathbf{H}}\left(\mathbf{Z}+\mathbf{Z}^{\mathbf{H}}\right) \hat{\mathbf{v}}_{\mathbf{n}}=\hat{\mathbf{v}}_{\mathbf{n}}^{\mathbf{H}} \mathbf{R} \hat{\mathbf{v}}_{\mathbf{n}}
$$

where $\mathbf{R}=S_{e} / 2 \Re(\mathbf{Z})$ is a real, symmetric, positive definite matrix, which is proportional to the radiation resistance matrix $\mathfrak{R}(\mathbf{Z})$. If the radiating surface is plane and in an infinite baffle, the terms of R may be calculated analytically using the Rayleigh integral (see Eq. (2)):

$$
Z_{M Q}=\frac{j \omega \rho_{f} S_{e}}{2 \pi r_{M Q}} e^{-j K r_{M Q}}, \quad M, Q=1 \ldots N
$$

where $r_{M Q}$ is the distance between the observation position and the elemental radiator (Fig. 2). Therefore, the matrix $\mathbf{R}$ is given by:

$$
\mathbf{R}(\omega)=\frac{\omega^{2} \rho_{f} s_{e}}{4 \pi c_{v}}\left[\begin{array}{cccc}
1 & \frac{\sin \left(K r_{12}\right)}{K r_{12}} & \ldots & \frac{\sin \left(K r_{1 N}\right)}{K r_{1 N}} \\
\frac{\sin \left(K r_{21}\right)}{K r_{21}} & 1 & \ldots & \frac{\sin \left(K r_{2 N}\right)}{K r_{2 N}} \\
\vdots & \vdots & \ddots & \vdots \\
\frac{\sin \left(K r_{N 1}\right)}{K r_{N 1}} & \frac{\sin \left(K r_{N 2}\right)}{K r_{N 2}} & \ldots & 1
\end{array}\right]
$$


This method can be applied to any plane surface in an infinite baffle, independently of the boundary conditions. It only requires the knowledge of the surface geometry, the characteristics of the fluid and the velocity field distribution. In this work, a finite element approach is used to evaluate this velocity field by using a sufficient number of discrete radiating elements according to the smallest wavelength to be observed.

\section{ELECTROMECHANICAL FINITE ELEMENT MODEL}

In this section, the general formulation of the equations that govern the mechanical and electrical state of an elastic structure equipped with piezoelectric patches is used to derive a finite element model [15-20]. Beforehand, the general problem is described and the main assumptions are recalled. Standard indicial notations are adopted throughout this section: subscripts $i, j, k, l$ denote the three-dimensional vectors and tensor components and repeated subscripts imply summation. In addition, a comma indicates a partial derivative.

\subsection{Hypotheses and Variational Formulation}

We consider the special case of an elastic structure, occupying a domain denoted $\Omega_{\mathrm{s}}$, is equipped with $P$ piezoelectric patches (Fig. (3)). Each piezoelectric patch has its upper and lower surfaces covered with a very thin electrode and can be slightly curved. The $p$-th patch, $p \in\{1, \cdots, P\}$, occupies a domain $\Omega^{(p)}$ such that $\left(\Omega_{s}, \Omega^{(1)}, \cdots, \Omega^{(p)}\right)$ is a partition of the whole domain $\Omega$. The domain $\Omega$ is subjected to prescribed body forces $f_{i}^{d}$ and the domain boundary, denoted $\partial \Omega$, is subjected to a prescribed displacement $u_{i}^{d}$ on a part $\Gamma_{u}$ and to a prescribed surface force density $t_{i}^{d}$ on the complementary part $\Gamma_{t}$ such that $\partial \Omega=\Gamma_{u} \cup \Gamma_{t}$.

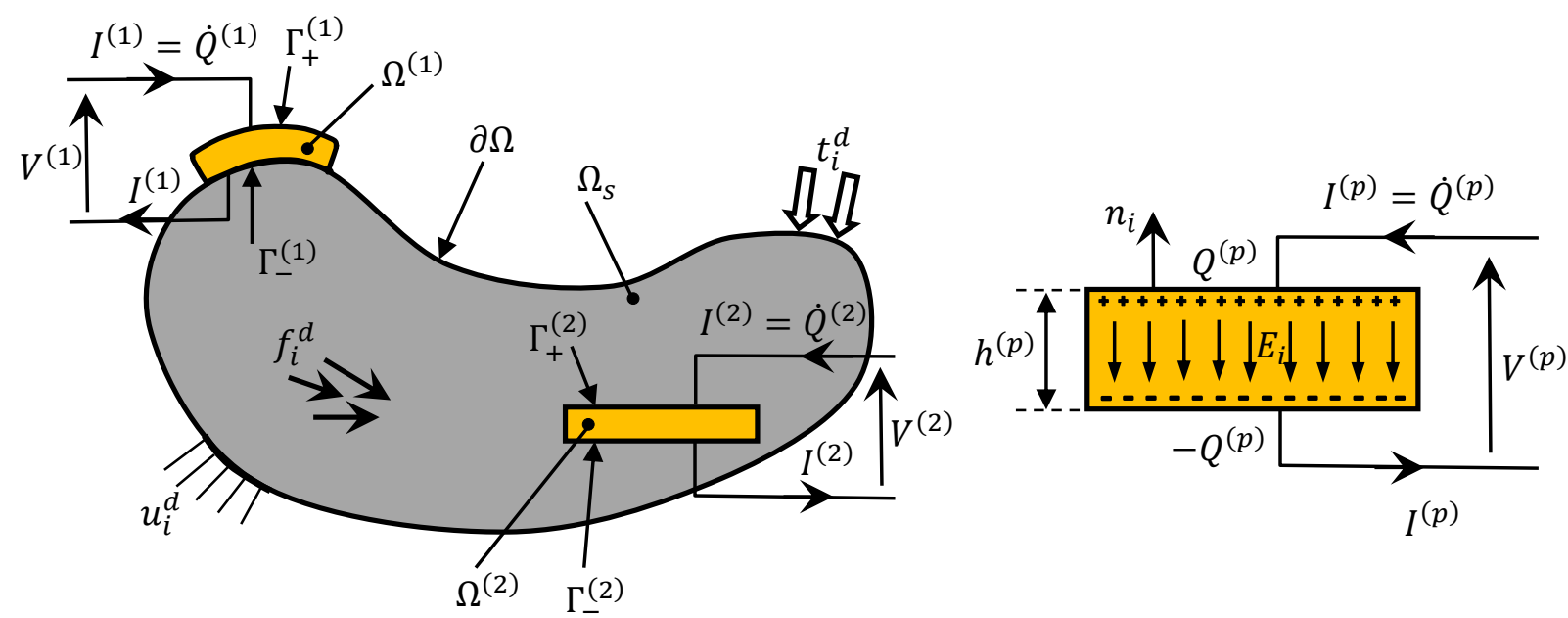

Figure 3. Left: an elastic structure with two piezoelectric patches. Right: the $p$-th piezoelectric patch submitted to the potential difference $V^{(p)}$, with its electrodes containing the free electric charges $Q^{(p)}$ and $-Q^{(p)}$ on their surface in contact with the patch [18]. 
A set of hypotheses, applicable to a wide spectrum of practical applications, can be used in order to obtain an efficient variational formulation of the problem. The main assumptions, detailed in [18], are summarized below.

- Only the piezoelectric patches are made of piezoelectric material. Consequently, the piezoelectric material constants $e_{i j k}$ vanish in $\Omega_{s}$. The electric displacement vector of component $D_{i}$ is neglected in the elastic domain $\Omega_{s}$, as compared to its value in any of the piezoelectric patches $\Omega^{(p)}$.

- The piezoelectric patches are thin with a constant thickness, denoted $h^{(p)}$ for the $p$-th patch, smaller than its characteristic longitudinal length. The thickness of the electrodes is much smaller than $h^{(p)}$ and is thus neglected.

- The piezoelectric patches are polarized in their transverse direction (i.e. the direction normal to the electrodes). Moreover, the electric field vector, of components $E_{k}$, is normal to the electrodes and uniform in the piezoelectric patch, so that for all $p \in$ $\{1, \ldots, P\}$

$$
E_{k}=-\frac{V^{(p)}}{h^{(p)}} n_{k} \text { in } \Omega^{(p)}
$$

where $V^{(p)}=\psi_{+}^{(p)}-\psi_{-}^{(p)}$ is the potential difference between the $p$-th patch upper and lower electrodes surfaces $\Gamma_{+}^{(p)}$ and $\Gamma_{-}^{(p)}$ and $n_{k}$ is the $k$-th component of the normal unit vector to the surface of the electrodes.

With appropriate initial conditions and considering the above hypotheses the variational formulation of the electromechanical problem is such that:

$$
\begin{gathered}
\int_{\Omega} c_{i j k l} \varepsilon_{k l}(u) \varepsilon_{i j}(\delta u) \mathrm{d} \Omega+\sum_{p=1}^{P} \frac{V^{(p)}}{h^{(p)}} \int_{\Omega^{(p)}} e_{k i j} n_{k} \varepsilon_{i j}(\delta u) \mathrm{d} \Omega+\int_{\Omega} \rho \frac{\partial^{2} u_{i}}{\partial t^{2}} \delta u_{i} \mathrm{~d} \Omega= \\
\int_{\Omega} f_{i}^{d} \delta u_{i} \mathrm{~d} \Omega+\int_{\Gamma_{t}} t_{i}^{d} \delta u_{i} \mathrm{~d} S \quad \forall \delta u_{i} \in C_{u}^{*}
\end{gathered}
$$

and

$-\sum_{p=1}^{P} \frac{\delta V^{(p)}}{h^{(p)}} \int_{\Omega^{(p)}} e_{i k l} \varepsilon_{k l}(u) n_{i} \mathrm{~d} \Omega+\sum_{p=1}^{P} \delta V^{(p)} C^{(p)} V^{(p)}=\sum_{p=1}^{P} \delta V^{(p)} Q^{(p)} \quad \forall \delta V \in \mathbb{R}$,

where $C^{(p)}=\epsilon_{33} S^{(p)} /\left(h^{(p)}\right)^{2}$ is the capacitance of the $p$-th piezoelectric patch $\left(\epsilon_{33}=\right.$ $\epsilon_{i k} n_{i} n_{k}$ being the piezoelectric permittivity in the direction normal to the electrodes and $S^{(p)}$ is the average area of the patch) and $Q^{(p)}$ the electric charge contained in the upper electrode of the $p$-th piezoelectric patch. For more details about the derivation of this original formulation, we refer the reader to [18]. It can be noted that we have defined $C_{u}$ as the space of sufficiently regular functions $u_{i}$ defined in the whole domain $\Omega$ and $C_{u}^{*}=\left\{u_{i} \in C_{u} \mid u_{i}=0\right.$ on $\left.\Gamma_{u}\right\}$.

\subsection{Finite Element Formulation}

Using any finite element procedure to discretize the mechanical part of Eq. (8) and (9) leads to introduce $\mathbf{U}$, the vector of nodal values of $u_{i}$. By introducing $\mathbf{Q}=\left(Q^{(1)} Q^{(2)} \ldots Q^{(P)}\right)^{\mathrm{T}}$ 
and $\mathrm{V}=\left(V^{(1)} V^{(2)} \ldots V^{(P)}\right)^{\mathrm{T}}$, the column vectors of electric charges and potential differences, one finally obtains the general finite element formulation of the electromechanical problem:

$$
\left(\begin{array}{cc}
M_{m} & 0 \\
0 & 0
\end{array}\right)\left(\begin{array}{l}
\ddot{U} \\
\ddot{V}
\end{array}\right)+\left(\begin{array}{cc}
K_{m} & K_{c} \\
-K_{c}^{T} & K_{e}
\end{array}\right)\left(\begin{array}{l}
U \\
V
\end{array}\right)=\left(\begin{array}{l}
F \\
Q
\end{array}\right)
$$

In the above equation, $\mathbf{M}_{\mathrm{m}}$ and $\mathbf{K}_{\mathrm{m}}$ are the mechanical mass and stiffness matrices, of size $N \times N$, where $N$ is the number of mechanical degrees of freedom. $\mathrm{K}_{\mathrm{c}}$ is the electromechanical coupling matrix, of size $N \times P . \mathrm{K}_{\mathbf{e}}=\operatorname{diag}\left(C^{(1)} \cdots C^{(P)}\right)$ is the diagonal matrix filled with the $P$ capacitances of the piezoelectric patches. $\mathbf{F}$ is the column vector of mechanical forcing, of length $N$.

The above discretized formulation equation is particularly adapted when the piezoelectric patches are shunted, that is to say, connected to a passive electrical network. In this case, neither $\mathbf{V}$ nor $\mathbf{Q}$ are prescribed by the electrical network but the latter imposes only a relation between them, that can be write as $\mathbf{f}(\ddot{\mathbf{V}}, \dot{\mathbf{V}}, \mathbf{V}, \ddot{\mathbf{Q}}, \dot{\mathbf{Q}}, \mathbf{Q})=\mathbf{0}$. This additional relation writes, in the cases of a resistive shunt composed of a resistance $R_{e}$ and a resonant shunt composed of a resistance $R_{e}$ and an inductance $L_{e}$ connected to the $p$-th patch:

$$
\begin{gathered}
V^{(p)}-R_{e} \dot{Q}^{(p)}=0 \quad \text { resistive shunt, } \\
V^{(p)}-L_{e} \ddot{Q}^{(p)}-R_{e} \dot{Q}^{(p)}=0 \quad \text { resonant shunt, }
\end{gathered}
$$

Depending on whether the patches are short-circuited $(\mathbf{V}=\mathbf{0})$ or in open-circuit $(\mathbf{Q}=\mathbf{0})$, the homogeneous problem associated to the discretized formulation (Eq. (10)) takes the following forms:

$$
\begin{gathered}
\mathrm{M}_{\mathrm{m}} \ddot{\mathrm{U}}+\mathrm{K}_{\mathrm{m}} \mathrm{U}=0 \text { short-circuit, } \\
\mathrm{M}_{\mathrm{m}} \ddot{\mathrm{U}}+\left(\mathrm{K}_{\mathrm{m}}+\mathrm{K}_{\mathrm{c}} \mathrm{K}_{\mathrm{e}}^{-1} \mathrm{~K}_{\mathrm{c}}^{\mathrm{T}}\right) \mathrm{U}=\mathbf{0} \text { open-circuit, }
\end{gathered}
$$

Therefore, one can notice the effect of open-circuit electromechanical coupling on the elastic structure appears as an added stiffness term $\mathrm{K}_{\mathrm{c}} \mathrm{K}_{\mathrm{e}}^{-1} \mathbf{K}_{\mathrm{c}}^{\mathrm{T}}$.

\subsection{Modal Expansion}

In this section, a reduced-order formulation of the discretized problem is derived by expanding the mechanical displacement unknowns vector onto the (i) short-circuit eigenmodes basis for the resistive shunt case and, (ii) coupled electromechanical basis for the resonant shunt case. Beforehand, the general finite element formulation in Eq. (10) will be rewrite in terms of the electrical unknown $\mathbf{Q}$. This $(\mathbf{U}, \mathbf{Q})$ formulation is well suited for shunting applications [18]. 


\subsubsection{Electromechanical problem with resistive shunt circuit}

In the case of the piezoelectric patches are coupled to the resistive shunt circuits and considering a mechanical viscous damping in the system, the general finite element formulation of the electromechanical problem becomes:

$$
\left(\begin{array}{cc}
\mathrm{M}_{\mathrm{m}} & 0 \\
\mathbf{0} & 0
\end{array}\right)\left(\begin{array}{c}
\ddot{\mathrm{U}} \\
\ddot{\mathrm{Q}}
\end{array}\right)+\left(\begin{array}{cc}
\mathrm{C}_{\mathrm{m}} & \mathbf{0} \\
\mathbf{0} & \mathrm{R}_{\mathrm{e}}
\end{array}\right)\left(\begin{array}{l}
\dot{\mathrm{U}} \\
\dot{\mathbf{Q}}
\end{array}\right)+\left(\begin{array}{cc}
\mathrm{K}_{\mathrm{m}}+\mathrm{K}_{\mathrm{c}} \mathrm{K}_{\mathrm{e}}^{-1} \mathrm{~K}_{\mathrm{c}}^{\mathrm{T}} & \mathrm{K}_{\mathrm{c}} \mathrm{K}_{\mathrm{e}}^{-1} \\
\mathrm{~K}_{\mathrm{e}}^{-1} \mathrm{~K}_{\mathrm{c}}^{\mathrm{T}} & \mathrm{K}_{\mathrm{e}}^{-1}
\end{array}\right)\left(\begin{array}{l}
\mathrm{U} \\
\mathbf{Q}
\end{array}\right)=\left(\begin{array}{l}
\mathrm{F} \\
0
\end{array}\right)
$$

where $\mathbf{C}_{\mathbf{m}}$ is the mechanical damping matrix and $\mathbf{R}_{\mathbf{e}}$ represent the diagonal electrical resistance matrix. In case the undamped free vibration is considered $(\mathbf{F}=\mathbf{0})$ and harmonic time dependency is assumed ( $\mathbf{U}=\widehat{\mathrm{U}} e^{j \omega t}$ and $\left.\mathbf{Q}=\widehat{\mathbf{Q}} e^{j \omega t}\right)$, Eq. (15) reduces to the short-circuit eigenvalue problem:

$$
-\omega^{2}\left(\begin{array}{cc}
\mathbf{M}_{\mathrm{m}} & 0 \\
\mathbf{0} & 0
\end{array}\right)\left(\begin{array}{l}
\widehat{\mathrm{U}} \\
\widehat{\mathbf{Q}}
\end{array}\right)+\left(\begin{array}{cc}
\mathrm{K}_{\mathrm{m}}+\mathrm{K}_{\mathrm{c}} \mathrm{K}_{\mathbf{e}}^{-1} \mathrm{~K}_{\mathrm{c}}^{\mathrm{T}} & \mathbf{K}_{\mathrm{c}} \mathrm{K}_{\mathbf{e}}^{-1} \\
\mathrm{~K}_{\mathbf{e}}^{-1} \mathbf{K}_{\mathrm{c}}^{\mathrm{T}} & \mathrm{K}_{\mathbf{e}}^{-1}
\end{array}\right)\left(\begin{array}{l}
\widehat{\mathrm{U}} \\
\widehat{\mathbf{Q}}
\end{array}\right)=\left(\begin{array}{l}
0 \\
0
\end{array}\right)
$$

Combining the second and first lines, we can easily see that the above discretized system correspond to the short-circuit eigenvalue problem $-\omega^{2} \mathbf{M}_{m} \widehat{\mathbf{U}}+\mathbf{K}_{m} \widehat{U}=\mathbf{0}$ which depends only on the mechanical properties of the system, i.e. the stiffness and mass of the structure with patches. The solution of this eigenvalue problem comprises $N$ natural angular frequencies $\omega_{\mathrm{i}}$ and corresponding eigenvectors $\widehat{\mathbf{U}}_{i}$ :

$$
\boldsymbol{\Omega}=\left[\begin{array}{cccc}
\omega_{1} & 0 & \cdots & 0 \\
0 & \omega_{2} & \cdots & 0 \\
\cdots & \vdots & \ddots & \vdots \\
0 & 0 & \cdots & \omega_{N}
\end{array}\right], \quad \boldsymbol{\Phi}=\left[\begin{array}{llll}
\widehat{\mathbf{U}}_{1} & \widehat{\mathbf{U}}_{2} & \cdots & \widehat{\mathbf{U}}_{N}
\end{array}\right]
$$

\subsubsection{Electromechanical problem with resonant shunt circuit}

In the case of the piezoelectric patches are coupled to the resonant shunt circuits and considering a mechanical viscous damping in the system, the general finite element formulation of the electromechanical problem becomes:

$$
\left(\begin{array}{cc}
\mathrm{M}_{\mathrm{m}} & \mathbf{0} \\
\mathbf{0} & \mathrm{L}_{\mathrm{e}}
\end{array}\right)\left(\begin{array}{c}
\ddot{\mathrm{U}} \\
\ddot{\mathrm{Q}}
\end{array}\right)+\left(\begin{array}{cc}
\mathrm{C}_{\mathrm{m}} & \mathbf{0} \\
\mathbf{0} & \mathrm{R}_{\mathrm{e}}
\end{array}\right)\left(\begin{array}{l}
\dot{U} \\
\dot{\mathbf{Q}}
\end{array}\right)+\left(\begin{array}{cc}
\mathrm{K}_{\mathrm{m}}+\mathrm{K}_{\mathrm{c}} \mathrm{K}_{\mathrm{e}}^{-1} \mathrm{~K}_{\mathrm{c}}^{\mathrm{T}} & \mathrm{K}_{\mathrm{c}} \mathrm{K}_{\mathrm{e}}^{-1} \\
\mathrm{~K}_{\mathrm{e}}^{-1} \mathrm{~K}_{\mathrm{c}}^{\mathrm{T}} & \mathrm{K}_{\mathrm{e}}^{-1}
\end{array}\right)\left(\begin{array}{l}
\mathrm{U} \\
\mathbf{Q}
\end{array}\right)=\left(\begin{array}{l}
\mathrm{F} \\
0
\end{array}\right)
$$

where $\mathbf{L}_{\mathbf{e}}$ represent the diagonal electrical inductance matrix. If we consider the undamped free vibration problem and if a harmonic time dependency is assumed, Eq. (18) reduces to the coupled electromechanical eigenvalue problem:

$$
-\omega^{2}\left(\begin{array}{cc}
\mathbf{M}_{\mathrm{m}} & \mathbf{0} \\
\mathbf{0} & \mathbf{L}_{\mathbf{e}}
\end{array}\right)\left(\begin{array}{c}
\widehat{\mathbf{U}} \\
\widehat{\mathbf{Q}}
\end{array}\right)+\left(\begin{array}{cc}
\mathrm{K}_{\mathrm{m}}+\mathrm{K}_{\mathrm{c}} \mathrm{K}_{\mathbf{e}}^{-1} \mathbf{K}_{\mathrm{c}}^{\mathrm{T}} & \mathbf{K}_{\mathrm{c}} \mathrm{K}_{\mathbf{e}}^{-1} \\
\mathrm{~K}_{\mathbf{e}}^{-1} \mathbf{K}_{\mathrm{c}}^{\mathrm{T}} & \mathbf{K}_{\mathbf{e}}^{-1}
\end{array}\right)\left(\begin{array}{l}
\widehat{U} \\
\widehat{\mathbf{Q}}
\end{array}\right)=\left(\begin{array}{l}
0 \\
0
\end{array}\right)
$$

As in the resistive shunt case, we can easily prove that the above discretized system do not correspond to short-circuit nor open-circuit eigenvalue problem. The solution of this ei- 
genvalue problem comprises $N+P$ natural angular frequencies $\omega_{i}$ and the corresponding eigenvectors $\Psi_{i}=\left[\widehat{\mathbf{U}}_{i} \widehat{\mathbf{Q}}_{i}\right]^{\mathrm{T}}$ :

$$
\boldsymbol{\Omega}=\left[\begin{array}{cccc}
\omega_{1} & 0 & \cdots & 0 \\
0 & \omega_{2} & \cdots & 0 \\
\cdots & \vdots & \ddots & \vdots \\
0 & 0 & \cdots & \omega_{N+P}
\end{array}\right], \quad \boldsymbol{\Psi}=\left[\begin{array}{llll}
\boldsymbol{\Psi}_{1} & \boldsymbol{\Psi}_{2} & \ldots & \boldsymbol{\Psi}_{N+P}
\end{array}\right]
$$

In order to reduce the mechanical part of the problem, we only use the mechanical eigenvectors portion, defined by:

$$
\boldsymbol{\Phi}=\left[\begin{array}{llll}
\widehat{\mathbf{U}}_{1} & \widehat{\mathbf{U}}_{2} & \cdots & \widehat{\mathbf{U}}_{N}
\end{array}\right]
$$

\subsubsection{Modal Expansion}

The mechanical displacement vector is sought as: $\mathrm{U}(t)=\sum_{i=1}^{N} \Phi_{i} q_{i}(t)$. As a result, the problem consists in solving the following system:

$$
\left(\begin{array}{cc}
\Phi^{\mathrm{T}} \mathrm{M}_{\mathrm{m}} \Phi & 0 \\
0 & \mathrm{X}
\end{array}\right)\left(\begin{array}{l}
\ddot{\mathrm{q}} \\
\ddot{\mathrm{Q}}
\end{array}\right)+\left(\begin{array}{cc}
\Phi^{\mathrm{T}} \mathrm{C}_{\mathrm{m}} \Phi & 0 \\
0 & \mathrm{R}_{\mathrm{e}}
\end{array}\right)\left(\begin{array}{l}
\dot{\mathrm{q}} \\
\dot{\mathbf{Q}}
\end{array}\right)+\left(\begin{array}{cc}
\Phi^{\mathrm{T}}\left(\mathrm{K}_{\mathrm{m}}+\mathrm{K}_{\mathrm{c}} \mathrm{K}_{\mathrm{e}}^{-1} \mathrm{~K}_{\mathrm{c}}^{\mathrm{T}}\right) \Phi & \Phi^{\mathrm{T}} \mathrm{K}_{\mathrm{c}} \mathrm{K}_{\mathrm{e}}^{-1} \\
\mathrm{~K}_{\mathrm{e}}^{-1} \mathrm{~K}_{\mathrm{c}}^{\mathrm{T}} \Phi & \mathrm{K}_{\mathrm{e}}^{-1}
\end{array}\right)\left(\begin{array}{c}
\mathrm{q} \\
\mathbf{Q}
\end{array}\right)=\left(\begin{array}{c}
\Phi^{\mathrm{T}} \mathrm{F} \\
0
\end{array}\right)
$$

where $\mathbf{X}=\mathbf{0}$ and $\boldsymbol{\Phi}$ is solution of Eq. (13) for resistive shunt circuits and $\mathbf{X}=\mathbf{L}_{\mathbf{e}}$ and $\boldsymbol{\Phi}$ is solution of Eq. (19) for the resonant shunt circuits.

\subsection{Modal Electromechanical Coupling Factors (MEMCF) and Shunt Electrical Com- ponents}

The well-known effective electromechanical modal coupling factor (EEMCF) characterizes the energy exchanges between the mechanical structure and the piezoelectric patches $[1,12,15,17-20,22]$. This parameter can be defined, for the system $i$-th mode, by

$$
K_{e f f, i}=\sqrt{\frac{\widehat{\omega}_{i}^{2}-\omega_{i}^{2}}{\omega_{i}^{2}}}
$$

where $\omega_{i}$ and $\widehat{\omega}_{i}$ are, respectively, the short-circuit and open-circuit $i$-th system natural frequencies (with all piezoelectric patches, respectively, short circuited or in open circuit).

The optimal values for the electrical parameters of both resistive and resonant shunts, in forced vibration condition, are presented in the table 1 . These classical results [19] depends only on (i) the natural frequency in short circuit of the considered vibration mode as well as its modal coupling factor (ii) the equivalent electrical blocked capacity of the patches. 
Table 1. Optimal electrical parameter values for the resistive and resonant shunt where $C_{e q}$ is the equivalent piezoelectric patches capacitance (see [19] for details).

\begin{tabular}{c|c}
\hline Shunt Circuit & Optimal Electrical Parameter \\
\hline Resistive & $R_{e}=\frac{1}{C_{e q} \omega_{i} \sqrt{1+\frac{K_{e f f, i}^{2}}{2}}}$ \\
\hline Resonant & $R_{e}=\frac{\sqrt{\frac{3}{2} K_{e f f, i}}}{C_{e q} \omega_{i} \sqrt{1+K_{e f f, i}^{2}}}$ \\
& $L_{e}=\frac{1}{C_{e q} \omega_{i}^{2}\left(1+K_{e f f, i}^{2}\right)}$ \\
\hline
\end{tabular}

\subsection{Electromechanical Finite Element Model Validation}

This section aims to validate the proposed electromechanical formulation (with one couple of electrical variables per patch as described in section 3) by comparison with a full $3 \mathrm{D}$ model (with one electric degree of freedom per node) $[2,5,11,18]$ and to show that the reduced order model is able to capture the main characteristics of the system dynamic behavior, especially in terms of attenuation. The considered example consist of a clamped-free beam-like structure made of aluminum material $\left(E=70 \mathrm{GPa}, \gamma=0.33, \rho=2700 \mathrm{~kg} / \mathrm{m}^{3}\right)$ with one piezoelectric patch made of PZT-4 material (see [11] for the properties). The geometrical dimensions are $175 \times 20 \times 2 \mathrm{~mm}^{3}$ for the structure and $50 \times 20 \mathrm{~mm}^{2}$ for the piezoelectric patch. The thickness of the piezoelectric patch varies between 1 to $5 \mathrm{~mm}$. To construct the two electromechanical models and solve the problems, the finite element code Nastran is used in association with Matlab software. Nastran is exploited here to create the elastic part of the models and to solve the complete electromechanical systems by means of DMAP language [14]. Matlab is used as a shell to manage Nastran and to construct the electric part of the models (see next section). The beam is modeled with quadratic quadrilateral shell elements (QUAD 8) and the piezoelectric patch is modeled using quadratic cubic solid elements (HEXA 20). A rigid connection between them is realized using RBE3 boundary element [14] (perfect bounding condition).

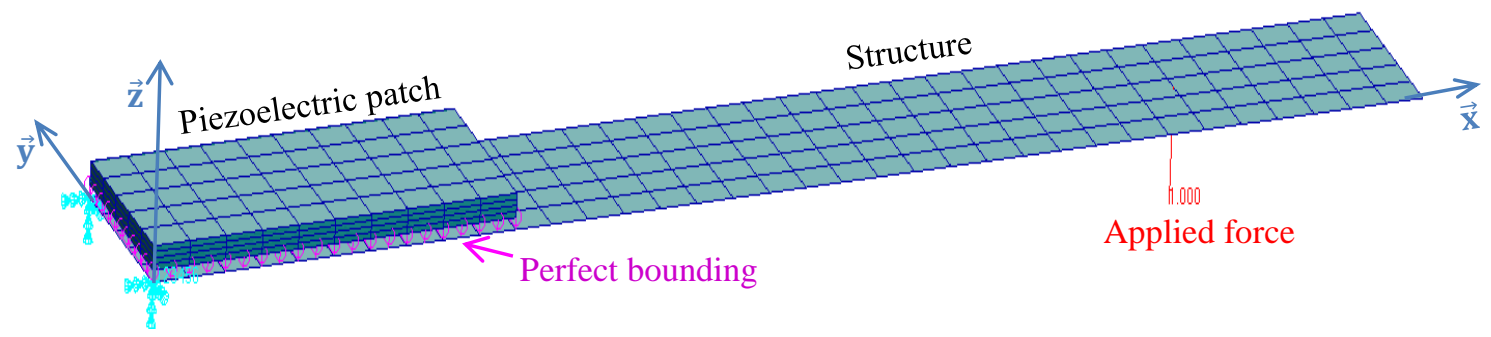

Figure 4. Clamped-free beam-like structure with one shunted piezoelectric patch: Finite element model into Nastran code. 
The frequency response functions (FRF) for a load case consisting of a unit point force in the $z$-direction and applied at co-ordinates $(x, y)=(145 \mathrm{~mm}, 10 \mathrm{~mm})$ is considered. Mechanical damping is introduced in the models through a structural damping coefficient $\eta=$ 0.02. The shunt circuit coupled to the piezoelectric patch was adjusted to attenuate the first vibration mode of the beam. The results are presented in Table 2 and Fig. 5. Table 2 shows the comparison of MEMCF between the proposed electromechanical formulation and the standard 3D electromechanical formulation. Fig. 5 show the transfer between the force point and the displacement at the location where this force is applied by using a resonant shunt circuit. It's important to note that:

- The results presented in Fig. 5 are obtained by using the same electrical parameters values (inductance and resistance) for the various electromechanical models;

- The reduced models were obtained using the formulation described in section 3 and with the ten first eigenmodes.

Table 2. EEMCF Comparison between the proposed electromechanical formulation and the standard 3D electromechanical formulation

\begin{tabular}{|c|c|c|c|c|}
\hline \multirow{4}{*}{$\begin{array}{c}\text { Vodal Electromechanical Coupling Factors (MEMCF) in } \% \\
\text { modes }\end{array}$} & $\begin{array}{c}\text { patch thickness=1mm } \\
\text { Standard } \\
\text { formulation }\end{array}$ & $\begin{array}{c}\text { Proposed } \\
\text { formulation }\end{array}$ & $\begin{array}{c}\text { Standard } \\
\text { formulation }\end{array}$ & $\begin{array}{c}\text { Proposed } \\
\text { formulation }\end{array}$ \\
\hline 1 & 12.12 & 12.21 & 2.73 & 2.85 \\
\hline 2 & 10.50 & 10.55 & 4.46 & 4.62 \\
\hline 3 & 0.00 & 0.00 & 0.00 & 0.00 \\
\hline 4 & 3.82 & 3.81 & 2.64 & 2.61 \\
\hline 5 & 0.00 & 0.00 & 0.00 & 0.00 \\
\hline 6 & 2.00 & 2.05 & 0.73 & 0.84 \\
\hline 7 & 0.00 & 0.00 & 0.00 & 0.00 \\
\hline 8 & 0.00 & 0.00 & 1.56 & 1.75 \\
\hline 9 & 4.35 & 4.42 & 0.00 & 0.00 \\
\hline 10 & 0.00 & 0.00 & 1.96 & 2.41 \\
\hline
\end{tabular}

We can see that the proposed formulation is in good agreement with the full 3D standard electromechanical model. Even if the difference between them increases with the thickness and size of piezoelectric patch, it remains very small for a patch with $5 \mathrm{~mm}$ of thickness. So we can conclude that our formulation is validated to compute electromechanical systems with added piezoelectric elements bounded on the host structure.

The results also validate the reduced models presented into the Section 3.3. 


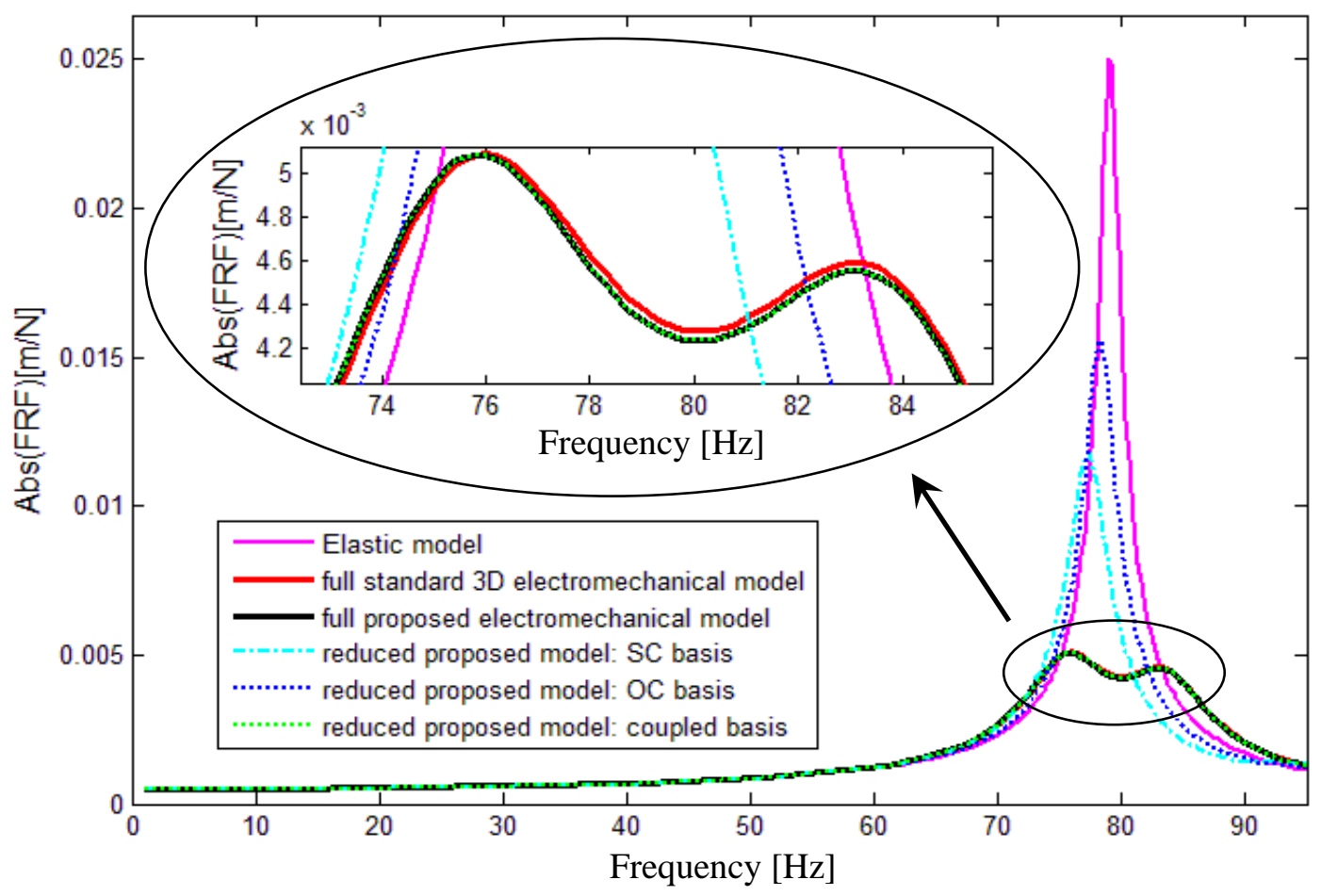

Figure 5. Transfer function between point force and beam response using a resonant shunt circuit and for patch thickness equal to $1 \mathrm{~mm}$.

\section{STRATEGY FOR THE COUPLING BETWEEN STRUCTURAL VIBRATION AND ACOUSTIC RADIATION}

This section presents the numerical strategy used to solve the vibroacoustic problem. As stated in section 2.1, the structural vibration and acoustic radiation problem can be solved independently due to the weak fluid-structure interaction. The commercial finite elements analysis program Nastran will be used in association with Matlab software.

\subsection{Numerical Strategy: Modeling of Noise and Vibration Reduction of Thin Structure}

As stated in section 1, the present work aims to propose an efficient approach able to predict the most radiating vibration modes of the structures and to attenuate these modes by using piezoelectric patches bonded on the structure and connected to resistive or resonant shunt circuits. To do that, two main steps are used: (i) determination the vibration modes of the structure (without piezoelectric patches) to be controlled and then, (ii) attenuation these modes by using shunted piezoelectric patches.

The first step is done by estimating the sound power radiated by the structure (without piezoelectric patches), in the frequency range of interest, using the elemental radiators method (see section 2.2).

Then, the next step consists in adding shunted piezoelectric patches on the structure to attenuate the selected vibration modes. The finite element formulation described in section 3 is used to solve the electromechanical problem. First, the modal analyses of the system short 
circuited and in open circuit are computed by resolving the Eq. (13) and (14), respectively. Then, the modal couplings factor (MEMCF) for the selected vibration modes and the optimal electrical parameters of the shunt circuits are obtained using Eq. (23) and the expressions presented in the table 1, respectively. Next, the vector of normal velocity on the structure surface is calculated solving the system described by Eq. (22). Finally, the sound power radiated is determined using the elemental radiators approach (section 2.2).

\subsection{Numerical Implementation: Modeling of Noise and Vibration Reduction of Thin Structure}

The above proceeding was numerical implemented according to the Fig. 6. Any modal analysis is done with the commercial finite element analysis program Nastran. It is a widely used software in the industry and is efficient for eigenmodes computation of large systems (several tens of thousands dofs). The Matlab software is exploited here as a shell manager, and as function to create and solving the electromechanical finite element model, and compute acoustic analysis. Throughout the procedure, much information is exchanged between Matlab, Patran and Nastran, for this reason, the programming language Awk is used because it allows reading and writing very fast.

\section{NUMERICAL EXAMPLES AND SENSITIVITY ANALYSES OF THE ELECTRO- VIBRO-ACOUSTIC PROBLEM}

This section aims to apply the general procedure introduced in Section 4 to a plate with one and several shunted piezoelectric patches. The main purposes of this section are to:

- Show the effectiveness of the proposed approach - reducing sound radiation from thin structures in the low frequency range by using shunted piezoelectric element;

- Perform sensitivity analyses concerning the piezoelectric patch (position and size) in order to highlight the role of the modal electromechanical coupling factor (EEMCF) on the optimization procedure of the shunt damping techniques for the reduction of sound radiation.

\subsection{Numerical examples}

To validate and illustrate the proposed approach, we consider a rectangular plate with two rectangular reinforcements perfectly bounded on its underside surface, both with $2 \mathrm{~mm}$ of thickness and made of aluminum material ( $E=70 \mathrm{GPa}, \gamma=0.33, \rho=2700 \mathrm{~kg} / \mathrm{m}^{3}$ ). The plate is in an infinite baffle and surround by the air $\left(\rho_{f}=1.2 \mathrm{Kg} / \mathrm{m}^{3}, c_{f}=340 \mathrm{~m} / \mathrm{s}\right)$. The complete problem description is given in Fig. 7. 


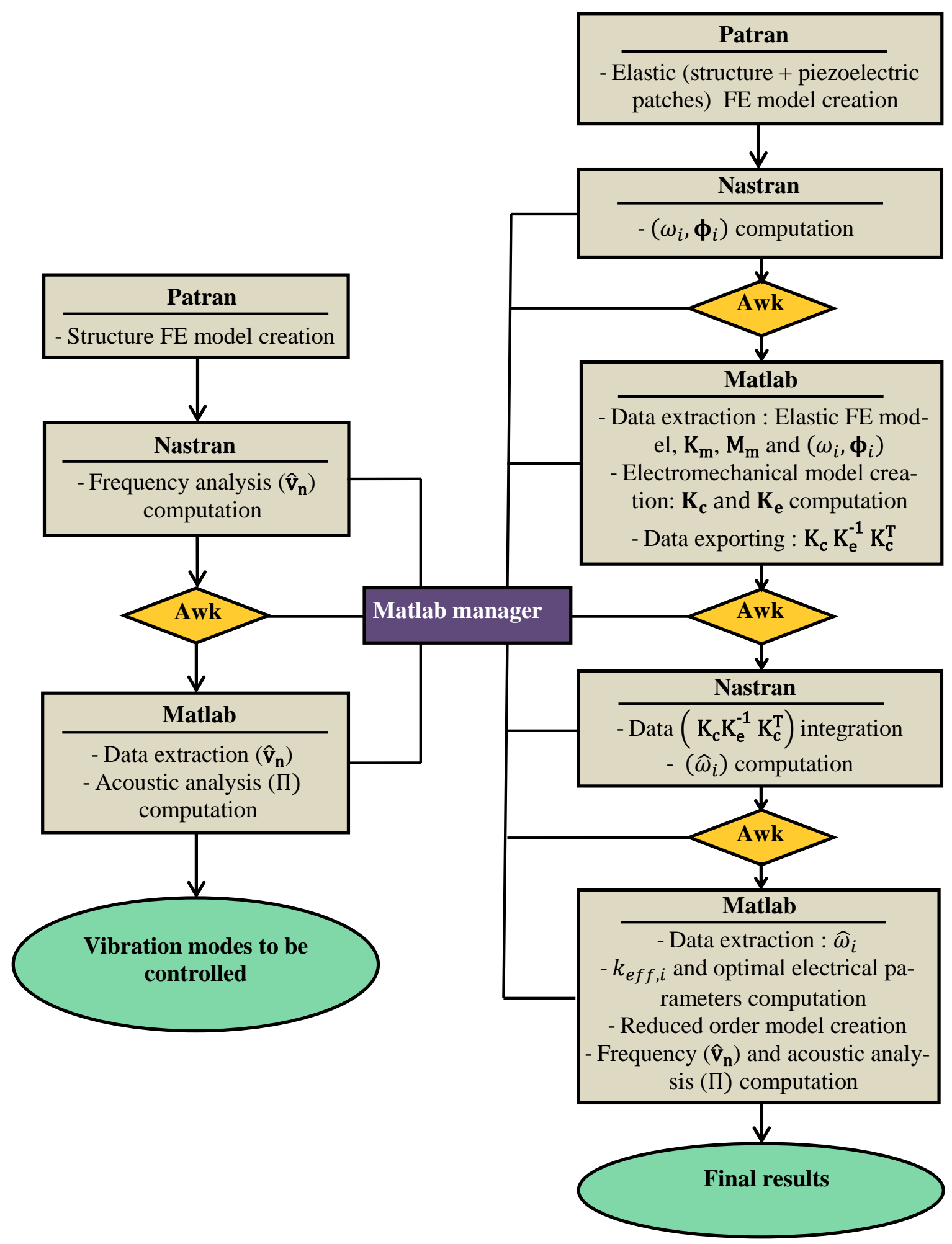

Figure 6. Left: First step - determination the vibration modes of the structure to be controlled. Right: Second step - attenuation of the select modes by using piezoelectric patches bonded on the structure and connected to resistive shunt circuits. For resonant shunt circuits, one additional modal analysis (section 3.3) needs to be performed before to do the reduced order model. This additional analysis is done by Nastran with Matlab management. 
The plate is excited by two transverse point forces of constant amplitude $(1 \mathrm{~N})$ whose positions are given in Fig. 7. It's modeled with 1536 linear quadrilateral shell elements with 4 nodes (Mindlin plate theory). The calculation of the eigenmodes of the system and the sound power radiated into the frequency band of interest $[0,430] \mathrm{Hz}$ (solid blue line curve in Fig. 8) show that vibration modes 1, 6 and 14 are the most radiating for the particular excitation. To attenuate these modes, three piezoelectric patches both with $1 \mathrm{~mm}$ of thickness and made of PIC151 material (see [18] for the properties) are bounded on the underside plate surface. The position and dimensions of these patches are given in Fig. 7. Patches 1,2 and 3 are connected to a shunt circuit to attenuate the plate vibration modes 1, 6 and 14, respectively. The system response is obtained by projection on the modal basis constructed from the first 40 eigenmodes of the structure (plate and shunted piezoelectric patches), according to section 3.3. Mechanical damping is introduced through a modal damping coefficient $\xi=0.05$ for all eigenmodes in the selected modal basis.

Fig. 8 shows the results for the resonant shunt case and Fig. 9 shows the results for the resistive shunt case. They demonstrate the efficiency of noise attenuation by using shunted piezoelectric elements. The attenuation obtained for the three modes remains around 12 to 15 $\mathrm{dB}$ using resonant shunts and 2 to $5 \mathrm{~dB}$ using resistive shunt. It is important to remember that:

- The level of vibration attenuation through the application of shunted piezoelectric elements depends on the modal damping coefficient (see [19]). For small damping coefficient, effective attenuation is large and, the effectiveness of attenuation decreases as the damping coefficient increases;

- The level vibration attenuation can be increased by optimizing the placement and size of piezoelectric patches [17, 20].
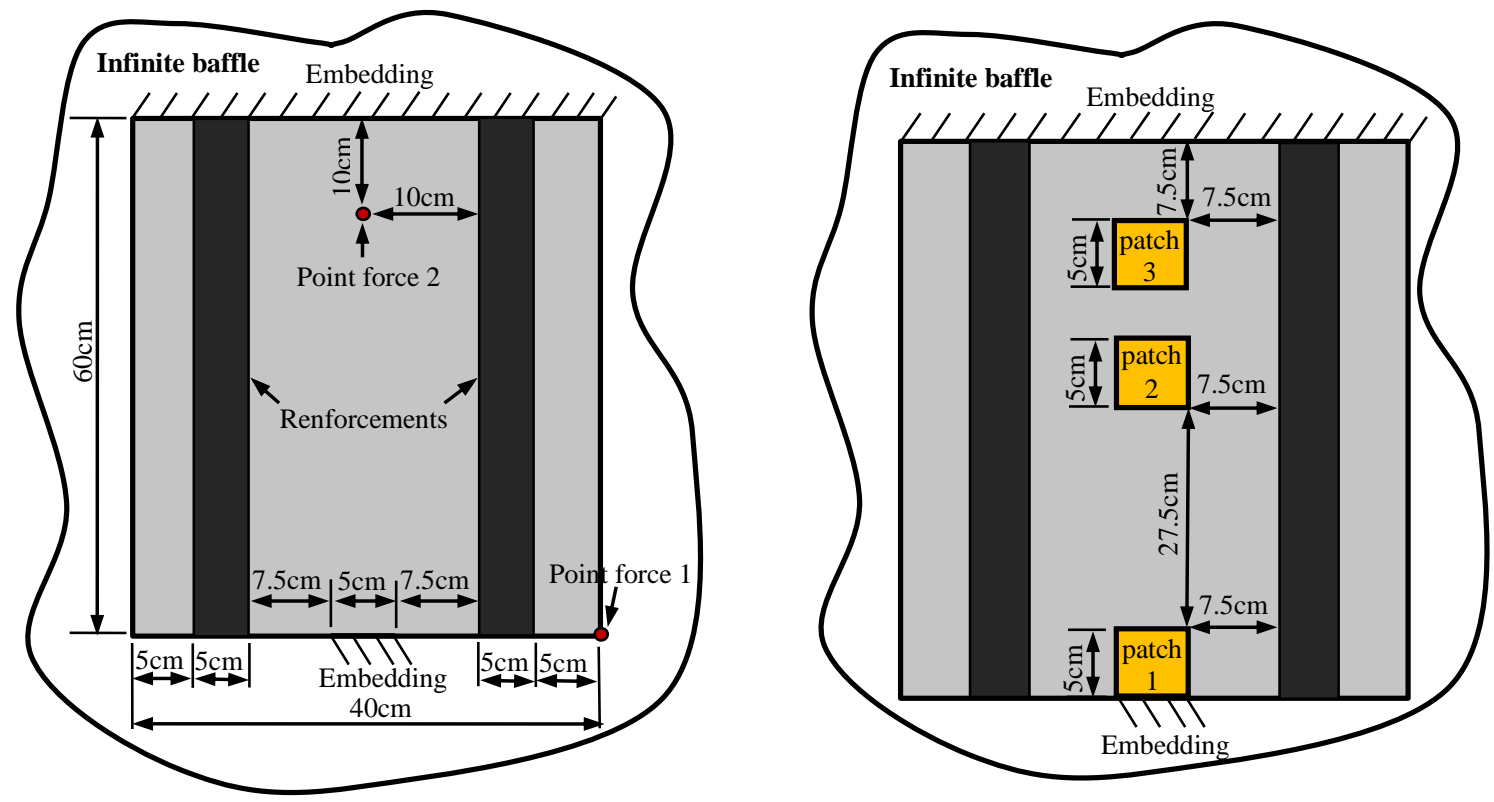

Figure 7. Left: Example layout without piezoelectric patches. Right: Example layout with piezoelectric patches. 


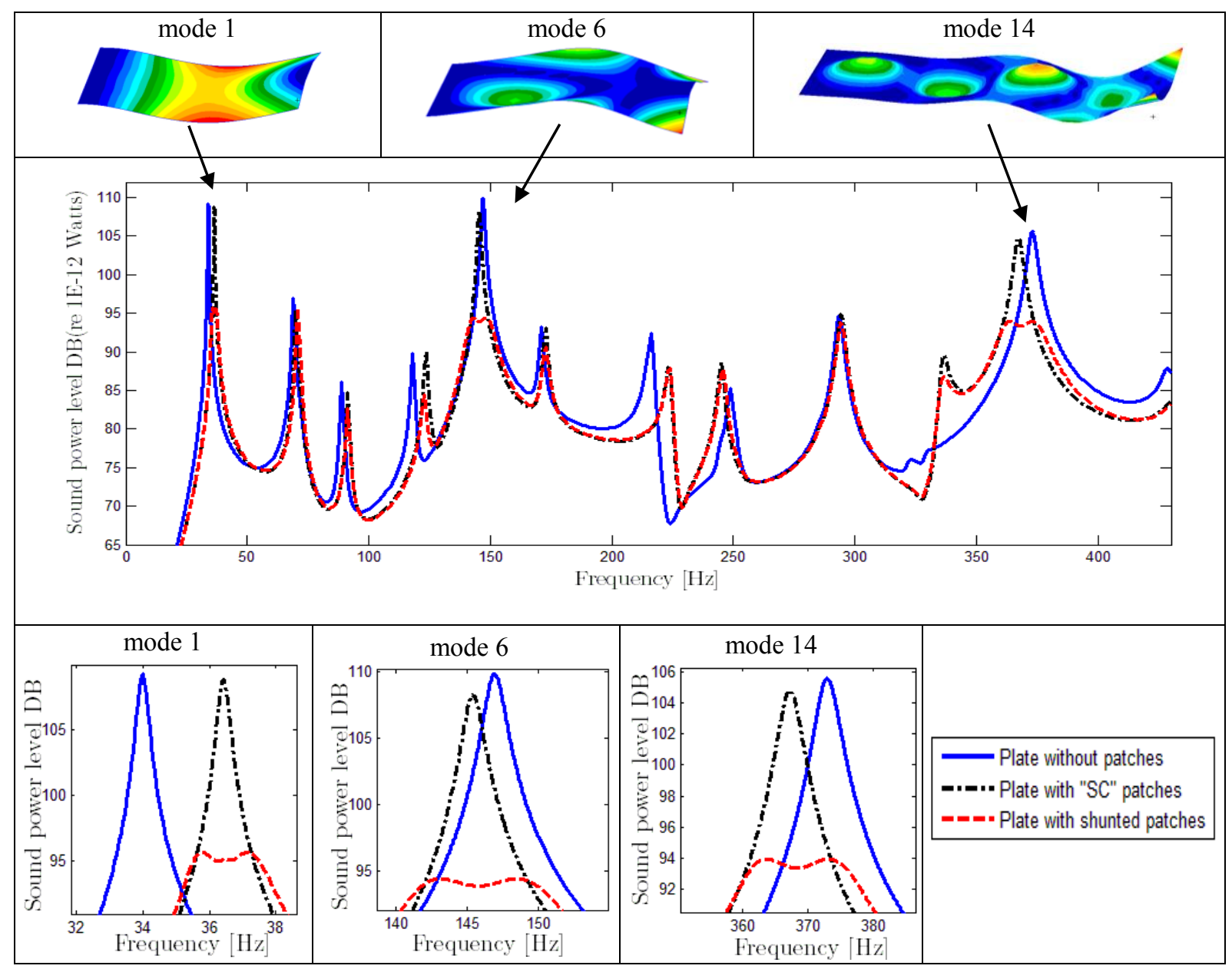

Figure 8. Sound power radiated for resonant shunt circuit case

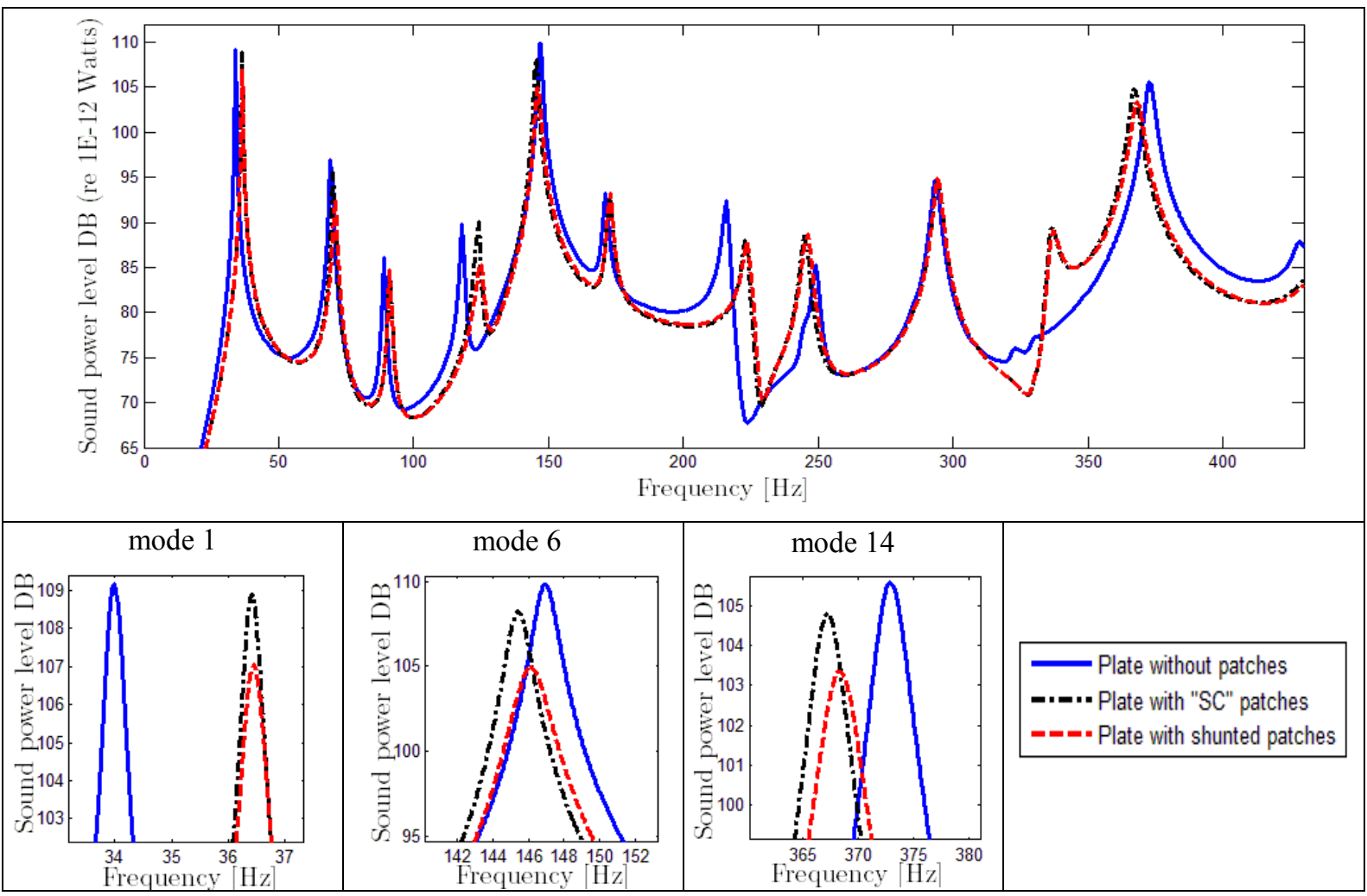

Figure 9. Sound power radiated for resistive shunt circuit case 


\subsection{Sensitivity Analyses for Electro-Vibro-Acoustic Optimisation}

It is well known that mechanical and electrical optimization can be realized separately $[17,19-20]$. The only parameter to maximize is the modal electromechanical coupling factors (MEMCF), which characterize the energy exchanges between the mechanical structure and the piezoelectric patches for a given mode. Since the optimal value of the electric circuit parameters are known as functions of the MEMCF and the system structural characteristics, they can be evaluated in a second step. Our objective is to optimize the placement and size of the piezoelectric patches for minimization of the acoustic power radiated. To achieve this, we consider the same rectangular plate as in the section 5.1, but with a single piezoelectric patch (same properties and dimensions of the patches presented in the Fig. 7). Moreover, we modified the placement of the patch on the plate surface. Fig. 10 shows the attenuation of the plate vibration mode 6 for two different positions of the piezoelectric patch shunted with a resistive shunt circuit (positions of the patch 1 and 2 of the Fig. 7).

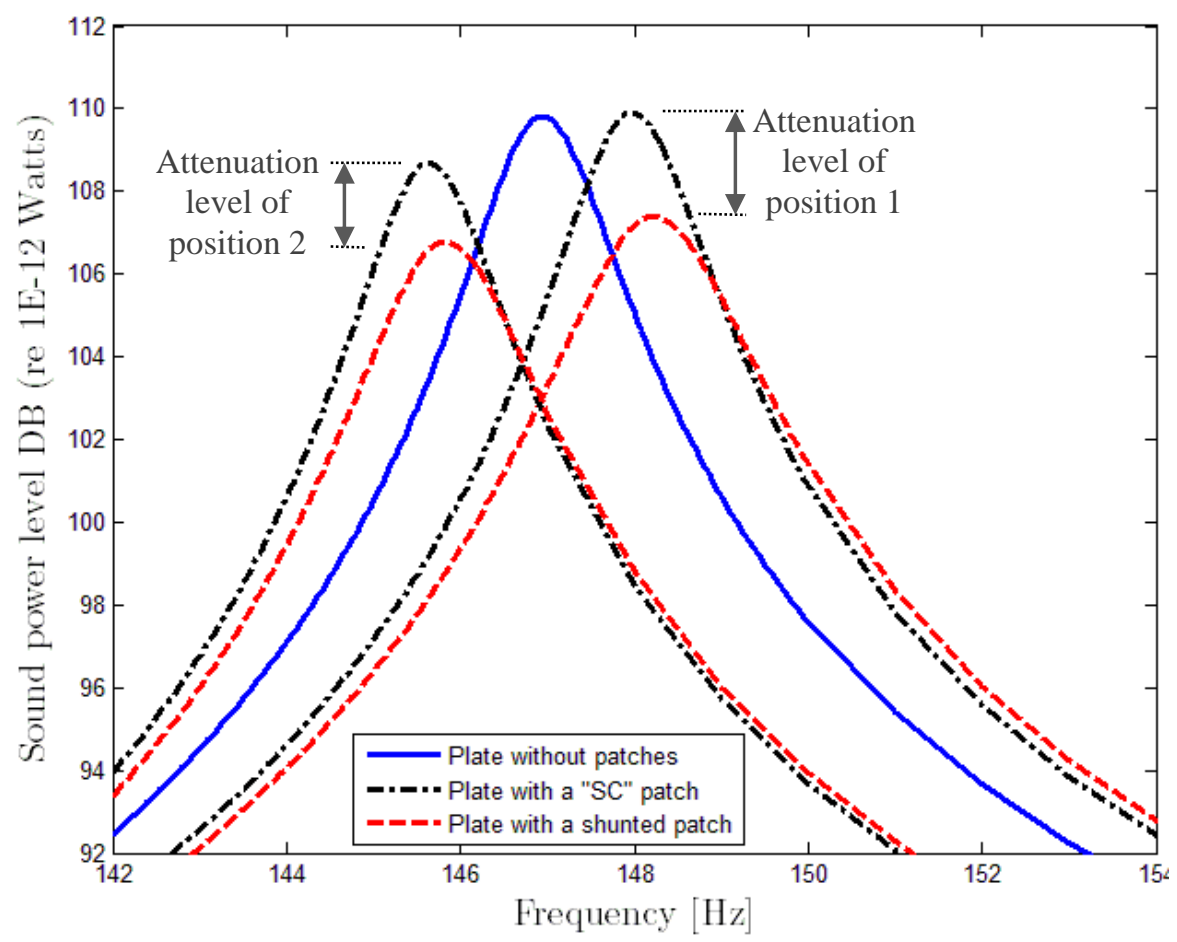

Figure 10. Sound power radiated for two positions of the piezoelectric patch shunted with a resistive shunt circuit

We can notice that the level of attenuation of the system with the piezoelectric patch on the position 1 (MEMCF $=6.91 \%)$ is larger than it is with the piezoelectric patch on the position 2 (MEMCF $=5.40 \%)$, but the level of the sound power radiated for the system with the piezoelectric patch on the position 2 is less than it is with the piezoelectric patch on the position 1 . This can be explaining by to the added mass and stiffness the piezoelectric patch. This example case brings us to conclude that the maximization of the MEMCF guarantee the maximization of the attenuation level but doesn't ensure the lowest level, especially for the resistive shunt type (low performance of attenuation). For systems with distributed patches 
throughout the structure, and for the case of the dynamic behavior is slightly modified by the piezoelectric elements (small patches), the maximization of this parameter guarantee the optimal placement and size of piezoelectric patches in an acoustic optimization procedure.

\section{CONCLUSIONS AND PERSPECTIVES}

In this study, an efficient numerical model for noise and vibration reduction of thin radiating planar structures in the low frequency range by using shunted piezoelectric elements has been proposed. First, the elemental radiators method used to solve the acoustic problem was recalled. Then, an original electromechanical finite element formulation, adapted to any elastic structures with surface-mounted piezoelectric patches, was presented and validated. Reduced order models of the electromechanical problem were then derived, by means of a modal expansion, taking account two types of electrical shunted circuits. These reduced models in association with the elemental radiators method were used to solve the electro-vibroacoustic problem. Preliminary examples are proposed in order to validate the approach. Future works concern the extension to (i) non planar thin structures taking into account the fluidstructure interaction, and (ii) multi-modal attenuation using a network of piezoelectric elements. Appropriate reduced order models will be also developed in order to optimize such coupled system.

\section{REFERENCES}

[1] ANSI/IEEE Standard 176-1987. “IEEE Standard on Piezoelectricity”. 1988.

[2] Benjeddou A., "Advances in piezoelectric finite element modeling of adaptive structural elements: a survey". Computers and Structures. 76(1-3), 347-363, 2000.

[3] Berkhoff A. P., "Sensor scheme design for active structural acoustic control". Journal of the Acoustical Society of America. 108(3), 1037-1045, 2000.

[4] Caruso G., "A critical analysis of electric shunt circuits employed in piezoelectric passive vibration damping”. Smart Materials and Structures. 10(5), 1059-1068, 2001.

[5] Collet M., Cunefare K.A., "Modal Synthesis and Dynamical Condensation Methods for Accurate Piezoelectric Systems Impedance Computation". Journal of Intelligent Material Systems and Structures. 19(11), 1251-1269, 2008.

[6] De Man P., "Controle actif du rayonnement acoustique des plaques : une approche à faible autorité". PhD thesis, Active Structures Laboratory of Université libre de Bruxelles.2004.

[7] dell'Isola F., Maurini C., Porfiri M., "Passive damping of beam vibrations through distributed electric networks and piezoelectric transducers: prototype design and experimental validation”. Smart Materials and Structures. 13 (2), 299-308, 2004. 
[8] Deü J.-F., Larbi W., Ohayon R., "Piezoelectric structural acoustic problems: Symmetric variational formulations and finite element results". Computer Methods in Applied Mechanics and Engineering. 197(19-20), 1715-1724, 2008.

[9] Elliott S. J., Johnson M. E., "Radiation modes and the active control of sound power". Journal of Acoustical Society of America. 94(4), 2194-2204, 1993.

[10] Filippi P. J. T., “Acoustique générale, SFA”. Les éditions de physique. 1994.

[11] Fernandes A., Pouget J., “Two-dimensional modelling of laminated piezoelectric composites: analysis and numerical results”. Journal of Thin-Walled Structures. 39, 3-22, 2001.

[12] Hagood N.W., Flotow A.V., "Damping of structural vibrations with piezoelectric materials and passive electrical networks". Journal of Sound and Vibration. 146(2), 243-268, 1991.

[13] Hollkamp J.J., "Multimodal passive vibration suppression with piezoelectric materials and resonant shunts". Journal of Intelligent Material Systems and Structures, 5 (1), 49-57, 1994.

[14] “MSC-NASTRAN 2010 Documentation”. MSC Software Corporation. 2010.

[15] Larbi W., Deü J.-F., Ciminello M., Ohayon R., "Structural-acoustic vibration reduction using switched shunt piezoelectric patches: A finite element analysis". Journal of Vibration and Acoustics. 132(5), 051006, 2010.

[16] Rosi G., Pouget J., dell'Isola F., "Control of sound radiation and transmission by a piezoelectric plate with an optimized resistive electrode". European Journal of Mechanics/A Solids. 29, 859-870, 2010.

[17] Sénéchal A., Thomas O., Deü J.-F., "Optimization of shunted piezoelectric patches for vibration reduction of complex structures - application to a turbojet fan blade". Proceedings of the ASME 2010 International Design Engineering Technical Conferences \& Computers and Information in Engineering Conference, IDETC/CIE 2010, Montreal, Canada. 2010.

[18] Thomas O., Deü J.-F., Ducarne J., "Vibrations of an elastic structure with shunted piezoelectric patches: efficient finite elements formulation and electromechanical couplings coefficients". International Journal for Numerical Methods in Engineering. 80(2), 235268, 2009.

[19] Thomas O., Ducarne J., Deü J.-F., "Performance of piezoelectric shunts for vibration reduction”. Smart Materials and Structures. 21(1), 015008, 2012. 
[20] Thomas O., Ducarne J., Deü J.-F., "Placement and dimension optimization of shunted piezoelectric patches for vibration reduction”. Journal of Sound and Vibration. 331 (14), 3286-3303, 2012.

[21] Trindade M.A., Benjeddou A., Ohayon R., "Finite element modeling of hybrid activepassive vibration damping of multilayer piezoelectric sandwich beams. Part 1: Formulation”. International Journal for Numerical Methods in Engineering. 51(7), 835-854, 2001.

[22] Trindade M.A., Benjeddou A., "Effective electromechanical coupling coefficients of piezoelectric adaptive structures: Critical evaluation and optimization". Mechanics of Advanced Materials and Structures. 16, 210-223, 2009.

[23] Trindade M.A., Maio C.E.B., "Multimodal passive vibration control of sandwich beams with shunted shear piezoelectric materials". Smart Materials and Structures. 17, 055015, 2008.

[24] Viana F.A.C., Steffen Jr V., "Multimodal vibration damping through piezoelectric patches and optimal resonant shunt circuits". Journal of the Brazilian Society of Mechanical Sciences. 28(3), 293-310, 2006.

[25] Wallace C.E., "Radiation Resistance of a Rectangular Panel, Journal of the Acoustical Society of America”. 51(3), 946-952, 1972. 